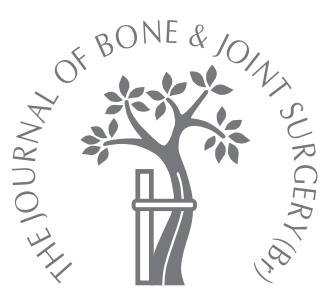
G. Bacci,
N. Fabbri,
A. Balladelli,
C. Forni,
E. Palmerini,
P. Picci

From the Department

of Musculoskeletal

Oncology, Istituti

Ortopedici Rizzoli,

Bologna, Italy

G. Bacci, MD,

Chemotherapist

N. Fabbri, MD, Orthopaedic

Surgeon

A. Balladelli, BA, Basic

Research

C. Forni, FRCS, Basic

Research

E. Palmerini, MD,

Chemotherapist

- P. Picci, MD, Pathologist

Istituti Ortopedici Rizzoli, Via

Pupilli 1, 40136 Bologna, Italy.

Correspondence should be sent to Dr G. Bacci; e-mail: gaetano.bacci@ior.it

C2006 British Editorial Society of Bone and Joint Surgery doi:10.1302/0301-620X.88B8. $17809 \$ 2.00$

$J$ Bone Joint Surg $[\mathrm{Br}]$ 2006;88-B:1071-5.

Received 24 February 2006; Accepted 12 April 2006

\title{
Treatment and prognosis for synchronous multifocal osteosarcoma in 42 patients
}

\begin{abstract}
Between 1986 and 2002, 42 patients with synchronous multifocal osteosarcoma were treated with two different protocols of neoadjuvant chemotherapy. When feasible, the primary and secondary tumours were excised as a combined procedure.
\end{abstract}

After initial chemotherapy 26 patients were excluded from simultaneous excision of all their secondary bone lesions as their disease was too advanced. In 12 patients only isolated excision of the primary lesion was possible. For 16 patients simultaneous operations were conducted to excise the primary and secondary lesions. This involved two supplementary sites in 15 patients and four additional sites in one patient. Of these, 15 attained remission but 12 relapsed and died (11 within two years). Three patients remained disease-free at five, six and 17 years. The histological response to pre-operative chemotherapy of the primary and secondary lesions was concordant in 13 of the 16 patients who underwent simultaneous operations at more than one site.

The prognosis for synchronous multifocal osteosarcoma remains poor despite combined chemotherapy and surgery. The homogeneous histological responses in a large proportion of the primary and secondary lesions implies that synchronous multifocal osteosarcoma tumours are not multicentric in origin, but probably represent bone-to-bone metastases from a single tumour.

Multifocal osteosarcoma is a distinct and unusual form of osteosarcoma in which, in addition to a single dominant tumour, other multiple smaller bone lesions are present. When the additional lesions are simultaneously present at diagnosis, the tumour is defined as a synchronous multifocal osteosarcoma. If smaller lesions appear at different intervals after treatment of the dominant lesion the term metachronous multifocal osteosarcoma is applied. ${ }^{1}$

Debate about the classification of these tumours (multicentric disease or multiple primary metastases) continues, ${ }^{2-4}$ although the prevailing opinion seems to be that synchronous multifocal osteosarcoma represents only one extreme of a vast spectrum of metastatic osteosarcoma. $^{5-7}$

In osteosarcoma of the extremities, adjuvant and neoadjuvant chemotherapy combined with surgery has dramatically improved the prognosis in patients with non-metastatic disease, ${ }^{8-12}$ and to a lesser extent has also improved the outcome of patients with lung metastases at presentation. ${ }^{13-16}$ In contrast, in synchronous multifocal osteosarcoma the role of chemotherapy combined with surgery at more than one site has not been investigated in a large series.

We report the results in 42 patients with synchronous multifocal osteosarcoma treated between 1986 and 2002 with two different protocols of neoadjuvant chemotherapy. The results of 23 of these patients, which have been previously reported, ${ }^{17,18}$ are updated.

\section{Patients and Methods}

From the 1154 cases of osteosarcoma treated at our institution between 1986 and 2002, 43 patients $(3.6 \%)$ had tumours of the extremities with bone metastases at presentation. Of these patients 17 also had lung metastases. One patient with lung metastases was transferred to another hospital for treatment, and the remaining 42 were treated according to the following protocol: primary chemotherapy, restaging, surgery (when feasible) on all neoplastic foci and adjuvant chemotherapy. Diagnosis and pathological evaluation. The diagnosis of osteosarcoma, established by clinical and radiological findings, was confirmed by histological examination of tissue obtained from an open or trocar biopsy, and from resected specimens, applying the principles of 
Table I. Neoadjuvant chemotherapy protocol

\begin{tabular}{|c|c|c|c|c|c|c|c|c|c|c|c|c|c|c|c|c|c|c|c|c|}
\hline \multicolumn{21}{|c|}{ IOR/OS-4* 20,21 } \\
\hline Treatment & M & P/A & M & I/P & I/A & Surgery & A & M & $\mathbf{P}$ & I & A & $\mathbf{M}$ & $\mathbf{P}$ & $\mathbf{I}$ & A & M & $\mathbf{P}$ & 1 & A & $\mathbf{M}$ \\
\hline Time (wks) & 0 & 1 & 4 & 5 & 8 & & 12 & 15 & 16 & 19 & 22 & 25 & 26 & 29 & 32 & 35 & 36 & 39 & 42 & 45 \\
\hline \multicolumn{21}{|c|}{ IOR/OS-5 $5^{\dagger 20,21}$} \\
\hline Treatment & $\mathbf{M}$ & P/A & I & M & P/A & I & Surgery & A & I & M & $\mathbf{P}$ & A & I & $\mathbf{M}$ & $\mathbf{P}$ & I & $\mathbf{M}$ & $\mathbf{P}$ & & \\
\hline Time (wks) & 0 & 1 & 4 & 6 & 7 & 10 & & 14 & 17 & 19 & 20 & 23 & 26 & 28 & 29 & 32 & 35 & 36 & & \\
\hline
\end{tabular}

* M, Methotrexate $12 \mathrm{~g} / \mathrm{m}^{2}$ in $4 \mathrm{~h}$ followed by CF rescue; P/A, Cisplatin $120 \mathrm{mg} / \mathrm{m}^{2}$ in $72 \mathrm{~h}$ infusion followed by adriamycin $60 \mathrm{mg} / \mathrm{m}^{2}$ in $8 \mathrm{~h}$ infusion; $\mathrm{I} / \mathrm{P}$, Ifosfamide $3 \mathrm{~g} / \mathrm{m}^{2} /$ day for 2 days in $1 \mathrm{~h}$ infusion followed by cisplatin $120 \mathrm{mg} / \mathrm{m}^{2}$ in $72 \mathrm{~h}$ infusion; l/A, Ifosfamide $3 \mathrm{~g} / \mathrm{m}^{2} / \mathrm{day}$ for $2 \mathrm{days}$ in $1 \mathrm{~h}$ infusion followed by adriamycin $60 \mathrm{mg} / \mathrm{m}^{2}$ in $8 \mathrm{~h}$ infusion; $\mathrm{P}$, cisplatin $120 \mathrm{mg} / \mathrm{m}^{2}$ in $72 \mathrm{~h}$ infusion; $\mathrm{A}$, adriamycin $45 \mathrm{mg} / \mathrm{m}^{2}$ day for $2 \mathrm{days}$ in $8 \mathrm{~h}$ infusion; $\mathrm{l}$, fosamide $2 \mathrm{~g} / \mathrm{m}^{2}$ day in $1 \mathrm{~h}$ infusion for 5 days

$\dagger \mathrm{M}$, Methotrexate $12 \mathrm{~g} / \mathrm{m}^{2}$ in $4 \mathrm{~h}$ followed by CF rescue; P/A, cisplatin $120 \mathrm{mg} / \mathrm{m}^{2}$ in $72 \mathrm{~h}$ infusion followed by adriamycin $75 \mathrm{mg} / \mathrm{m}^{2}$ in $6 \mathrm{~h}$ infusion; $\mathrm{I} / \mathrm{P}$, Ifosfamide $3 \mathrm{~g} / \mathrm{m}^{2} /$ day for 2 days in $1 \mathrm{~h}$ infusion followed by cisplatin $120 \mathrm{mg} / \mathrm{m}^{2}$ in $72 \mathrm{~h}$ infusion; I/A, Ifosfamide $3 \mathrm{~g} / \mathrm{m}^{2} / \mathrm{day}$ for 2 days in $1 \mathrm{~h}$ infusion followed by adriamycin $60 \mathrm{mg} / \mathrm{m}^{2}$ in $8 \mathrm{~h}$ infusion; $\mathrm{A}$, adriamycin $90 \mathrm{mg} / \mathrm{m}^{2}$ in $24 \mathrm{~h}$ continuous infusion; $\mathrm{P}$, cisplatin $120 \mathrm{mg} / \mathrm{m}^{2}$ in $72 \mathrm{~h}$ continuous infusion; I, Ifosamide $15 \mathrm{~g} / \mathrm{m}^{2}$ day in $120 \mathrm{~h}$ continuous infusion

Fletcher et $\mathrm{al}^{19}$ and classifying the tumour as classic, telangiectatic or small cell. On the basis of predominant cells and intercellular material, classic osteosarcoma was subclassified as osteoblastic, fibroblastic or chondroblastic. When two of the different types of osteoid, cartilage and fibrous tissue were present in almost the same proportions, the tumour was classified as conventional mixed osteosarcoma.

Pre-operative evaluation. The primary tumour was evaluated with standard radiographs, CT and technetium99MDP bone scans. Approximately half also underwent MR scanning. Bone metastases were investigated by total body scans. If this examination revealed other bone neoplastic foci, these were confirmed by CT and/or MRI. A CT scan of the chest was performed in order to exclude lung metastases. All these tests were repeated after the initial chemotherapy.

Chemotherapy. This was performed according to two different protocols (IOR/OS-4 and IOR/OS-5), reported in detail elsewhere ${ }^{20,21}$ and summarised in Table I. Haematopoietic, renal and liver function was strictly monitored. No dose reduction was accepted by the protocols. If the white blood cell count was less than $1 \times 10^{9} / 1$ and/or the platelet count less than $100 \times 10^{9} / 1$ before each planned cycle of chemotherapy, treatment was delayed until the blood count had recovered.

Surgery and evaluation of response to chemotherapy. When feasible, surgery of the primary and secondary bone lesions was programmed according to location and extension of the tumour and/or the involvement of neurovascular structures. For the primary tumour surgical treatment was amputation, rotationplasty or limb salvage. When undertaking limb-salvage procedures, we either used a prosthesis or a vascularised fibula graft, combined with either allograft or autograft. The surgical treatment of secondary bone tumours depended on the site and extent of the tumour. Accordingly, either resection, limb salvage or amputation was performed.

After surgery, the surgeons and pathologists reviewed the gross specimens together to determine the surgical margins according to Enneking's classification. ${ }^{22}$ The response to pre-operative chemotherapy of the primary and secondary tumours was graded as good $190 \%$ or more tumour necrosis) or poor (less than $90 \%$ tumour necrosis), as described by Picci et al. ${ }^{23}$

Post-relapse treatment and outcome. Treatment for metastatic lesions and/or local recurrence in relapsed patients was not standardised, but performed on an individual basis according to the location, the number of recurrences and disease-free interval. None the less, the key point of treatment was generally complete excision of metastases and/or local recurrence whenever possible.

Follow-up procedures. During treatment and after finishing chemotherapy the patients were reviewed every two months for five years for metastases or local recurrence. ${ }^{20}$ They were reviewed for five years at intervals previously reported. ${ }^{20}$ Subsequent information was obtained by telephone interview.

Statistical analysis. The main focus of this study was eventfree survival. Recurrence, and death from toxicity were considered to be adverse effects. Overall survival was also studied, although the data should be considered with caution because we do not know the exact treatment received by patients who were moved to another hospital for any part of their treatment. For the patients in remission, event-free survival was calculated from the first day of pre-operative chemotherapy to the first adverse event or to the most recent follow-up examination. Overall survival was calculated from the first day of chemotherapy until death, or the last follow-up. The Kaplan-Meier method was used to calculate survival curves and the log-rank test was used to compare the results.

\section{Results}

The demographic data of the 42 patients studied are summarised in Table II. In 40 patients the symptoms were related to the primary tumour, with the other asymptomatic bone lesions only detected during staging procedures. Only two patients, both with spinal secondary lesions, were symptomatic at presentation. Serum alkaline phosphatase was normal in only five patients and raised in 37 . The mean volume of the primary tumour was $516 \mathrm{ml}$ (178 to 1200). 
Table II. Demographic details of the 42 patients and number of patients in remission

\begin{tabular}{|c|c|c|c|}
\hline & $\begin{array}{l}\text { Number of } \\
\text { patients }\end{array}$ & $\begin{array}{l}\text { Number of } \\
\text { patients in } \\
\text { remission }\end{array}$ & $\mathbf{p}$ \\
\hline \multicolumn{4}{|l|}{ Gender } \\
\hline Male:female & $24: 18$ & $11: 4$ & 0.2 \\
\hline \multicolumn{4}{|l|}{ Age } \\
\hline Mean & 16 (3 to 62$)$ & & \\
\hline$\leq 15 \mathrm{yrs}$ & 23 & 8 & 0.85 \\
\hline$>15$ yrs & 19 & 7 & \\
\hline \multicolumn{4}{|l|}{ Tumour site } \\
\hline Only bone lesions & 25 & 10 & 0.70 \\
\hline Bone and lung lesions & 17 & 5 & \\
\hline \multicolumn{4}{|c|}{ Number of additional bone lesions } \\
\hline One & 13 & 10 & 0.001 \\
\hline Two & 8 & 4 & \\
\hline Three & 4 & 1 & \\
\hline Four & 10 & 0 & \\
\hline Five & 6 & 0 & \\
\hline$>$ five & 1 & 0 & \\
\hline \multicolumn{4}{|l|}{ Primary tumour site } \\
\hline Femur & 26 & 10 & 0.88 \\
\hline Tibia & 6 & 2 & \\
\hline Humerus & 10 & 3 & \\
\hline \multicolumn{4}{|l|}{ Serum alkaline phosphatase } \\
\hline Normal & 5 & 4 & 0.06 \\
\hline Elevated & 21 & 6 & \\
\hline Very elevated & 16 & 5 & \\
\hline \multicolumn{4}{|l|}{ Lactate dehydrogenase } \\
\hline Normal & 16 & 5 & 0.88 \\
\hline Elevated & 26 & 10 & \\
\hline \multicolumn{4}{|l|}{ Primary tumour volume } \\
\hline$\leq 300 \mathrm{ml}$ & 9 & 4 & 0.82 \\
\hline$>300 \mathrm{ml}$ & 33 & 11 & \\
\hline \multicolumn{4}{|l|}{ Histology } \\
\hline Osteoblastic & 35 & 12 & 0.25 \\
\hline Chondroblastic & 2 & 0 & \\
\hline Fibroblastic & 2 & 1 & \\
\hline Unclassified & 2 & 2 & \\
\hline Small round cell & 1 & 0 & \\
\hline
\end{tabular}

The mean time between onset of symptoms and final diagnosis was 1.9 months ( 1 to 7 ).

Number and site of secondary bone lesions. The total number of secondary bone lesions was 117 with a mean per patient of 2.8 ( 1 to 6 ). The absolute numbers are shown in Table II. The sites of these secondary lesions were the spine (26), ribs (25), pelvis (25), other extremity bones (27), scapula (7), clavicle (3), sternum (2) and skull (2). In all but the two symptomatic patients, the bone lesions were detected by bone scanning and confirmed by CT and/or MRI. Of the 117 bone lesions 94 were also evident on plain radiographs. Clinical, radiological and laboratory response to primary chemotherapy. Following the pre-operative chemotherapy in 38 patients there was an improvement in pain, a reduc-
Table III. Analysis of patients who entered remission according to treatment

\begin{tabular}{|c|c|c|c|}
\hline & $\begin{array}{l}\text { Number of } \\
\text { patients }\end{array}$ & $\begin{array}{l}\text { Number of } \\
\text { patients in } \\
\text { remission }\end{array}$ & $\mathbf{p}$ \\
\hline \multicolumn{4}{|c|}{ Surgery primary lesion } \\
\hline Amputation & 4 & 1 & 0.001 \\
\hline Limb salvage & 23 & 14 & \\
\hline Rotationplasty & 1 & 0 & \\
\hline None & 14 & 0 & \\
\hline \multicolumn{4}{|c|}{ Surgery secondary lesion } \\
\hline None & 26 & 0 & 0.0001 \\
\hline Two sites & 15 & 14 & \\
\hline Four sites & 1 & 1 & \\
\hline \multicolumn{4}{|c|}{ Surgical margins (primary lesions) } \\
\hline Wide & 26 & 14 & 0.52 \\
\hline Marginal & 2 & 1 & \\
\hline \multicolumn{4}{|c|}{ Chemotherapy protocol } \\
\hline IOR/OS-4 $4^{20,21}$ & 18 & 8 & 0.48 \\
\hline IOR/OS-5 $5^{20,21}$ & 24 & 7 & \\
\hline \multicolumn{4}{|c|}{ Histological response } \\
\hline Poor & 18 & 8 & 0.36 \\
\hline Good & 10 & 7 & \\
\hline
\end{tabular}

tion in swelling around the primary tumour and an increase in available movement where it had been previously restricted. Radiologically, in 32 patients the primary tumour increased in density, with better definition of its margins following the first course of chemotherapy. In six patients a reduction in tumour size of $10 \%$ to $20 \%$ was also observed. In six patients no significant changes were seen, and in four the tumour progressed.

No significant radiological changes were seen in synchronous bone lesions, but an increased radiodensity was observed in $55 \%$ of secondary foci.

In the 15 patients who also had lung metastases these resolved in two patients and in eight they reduced in size by $20 \%$ to $70 \%$. In three patients they remained unchanged, and in two they progressed during pre-operative treatment. Generally the lung metastases remained increased in density. A lung metastasis emerged during the pre-operative chemotherapy in one patient who had only had bone lesions at initial presentation.

Of the 37 patients with raised serum alkaline phosphatase levels it returned to normal in 20 and was lowered in the remaining 17 even when local or systemic progression continued during the pre-operative treatment.

Surgical treatment. The type of surgery for the primary lesions as well as the number of operated secondary foci are shown in Table III. In 26 patients, even after the induction chemotherapy, it was not possible to operate on all the secondary tumour sites involved (including lung metastases). In 12 patients with inoperable secondary lesions only the primary lesion was operated on, with amputation being the 
only option in three cases with large ulcerating tumours that had failed to respond to chemotherapy. Limb salvage was performed in eight patients (five with prostheses and three with a vascularised fibular graft with allograft and autograft) and rotationplasty in one.

The remaining 16 patients underwent simultaneous operations on more than one bone. In 15 patients this involved two bones and in one patient four bones. Three of the 15 patients who received simultaneous operations on two bones had wedge resection of one, three and five metastatic nodules involving their lungs, under the same anaesthetic. Of these 16 patients, 15 had complete remission, but in the remaining patient it was not possible to remove all the metastatic pulmonary nodules. This patient and those not suitable for operative treatment following the pre-operative chemotherapy were transferred to other institutions, where they were treated with radiotherapy, other chemotherapy or palliative therapy only. All these patients died at a mean of 8 months (4 to 19) after the diagnosis was established. Therefore, of the 42 patients who entered the study, only 15 reached disease-free status, and 27 never entered remission.

Histological response to pre-operative chemotherapy. The histological response to pre-operative chemotherapy of the 28 primary tumours operated on was good in ten patients and poor in 18. In the 19 secondary lesions which were excised the histological response to chemotherapy was good in eight and poor in 11 . Of the 16 patients who had combined resection of primary and secondary lesions the histological response was good at both sites in five patients, poor at both sites in eight, and good at the primary site but poor at the secondary site in three. In summary, there was concordance of response between the primary and secondary lesions in 13 of 16 cases. The patient who had combined resection of three secondary lesions had a good histological response at all sites.

Outcome of patients in remission. Of the 15 patients who entered remission, three have remained free of disease at five, seven and 17 years after treatment. These three patients, aged eight, 12 and 12 years old at diagnosis, had only two sites involved (femur-rib, femur-contralateral tibia, humerus-rib). Their primary tumours were treated by limb salvage with wide resection margins and had a good histological response to chemotherapy. At presentation their alkaline phosphatase level was normal in one case and raised in two. Two of these patients were treated with the IOR/OS-5 protocol and one with the IOR/OS-4 protocol.

The remaining 12 patients relapsed at a mean of 15.4 months ( 3 to 60 ) after surgery. The first relapses were new bone metastases in four cases, lung metastases in two, bone metastases and local recurrence in three, and lung and bone metastases in three. Of these patients, despite further treatment, ten died from the consequences of the tumour and two from toxicity of the second-line chemotherapy. The mean interval between surgery and death in these patients was 23 months (9 to 72 ).
As shown in Table II, the favourable factors for eventfree survival were the number of additional bone lesions and, to a lesser extent, the male gender.

\section{Discussion}

Synchronous multifocal osteosarcoma, originally described by Silverman in $1936,{ }^{24}$ is a rare variety of osteosarcoma with multiple skeletal sites involved at presentation. Usually patients have pain and swelling in only one site (dominant lesion), whereas other bone locations (secondary lesions) are asymptomatic and found only during initial investigation with bone scintigraphy. Radiological examination generally shows a large, principal lesion similar to classic radiolucent osteosarcoma, associated with other smaller bone lesions scattered throughout the skeleton.

Various classifications of synchronous multifocal osteosarcoma have been proposed, ${ }^{2,5,25}$ and whether it represents an early bone-seeking metastatic spread or a tumour with multicentric origin is still debated. The reported incidence of synchronous multifocal osteosarcoma ranges from $0.4 \%{ }^{26}$ to $4.2 \%{ }^{4}$ In our series the incidence was $3.4 \%$ (2.2\% excluding patients who also had lung metastases).

Synchronous multifocal osteosarcoma has been generally considered fatal within a few months, ${ }^{1,27}$ but this information hails from the pre-chemotherapy era. In osteosarcoma of the extremities without detectable metastases at presentation, combined chemotherapy and surgery has been thoroughly studied and shown to improve the success of treatment to $60 \%$ to $70 \%$, compared to $10 \%$ to $20 \%$ in patients treated by surgery alone..$^{8-12}$ Additionally, in patients with pulmonary metastases at presentation ${ }^{13-16}$ and those with localised disease who develop isolated lung metastases after adjuvant or neoadjuvant treatment, ${ }^{28}$ resection of the pulmonary metastases with or without second-line chemotherapy has led to a cure rate of about $25 \%$ and a five-year overall survival of $50 \%$.

In synchronous multifocal osteosarcoma the role of chemotherapy combined with surgery has not been investigated in a large series. Our results indicate that the prognosis for synchronous multifocal osteosarcoma remains extremely poor, despite combined chemotherapy and surgery. Only three of our patients remained free of disease for five or more years, whereas the rest died, $35(83 \%)$ within two years of diagnosis.

It remains unclear whether synchronous multifocal osteosarcoma is a real multicentric tumour or the simple expression of an aggressive tumour with early systemic bone dissemination. In neoadjuvant treatment of nonmetastatic osteosarcoma of the extremities the histological response to chemotherapy is strictly correlated to prognosis. $^{8-12,16,17}$ It is possible that the primary tumour and undetectable micrometastases share cells with the same chemosensitivity. In our study 13 of 16 patients who had simultaneous operations on two or more bones had the same histological response (good/good or poor/poor) for both the primary and the secondary lesions. This implies 
that the primary and secondary tumours have the same chemosensitivity and supports the theory that different bone lesions in synchronous multifocal osteosarcoma are not multicentric in origin but are more likely bone-to-bone metastases of a single tumour.

No benefits in any form have been received or will be received from a commercial party related directly or indirectly to the subject of this article.

\section{References}

1. Mirra JA. Bone tumors: clinical, radiological and pathologic correlations. Vol 2. London: Lea \& Fabiger, 1989:344-50.

2. Amstutz HC. Multiple osteogenic sarcomata: metastatic or multicentric: report of two cases and review of literature. Cancer 1969;24:923-31.

3. Jones RD, Reid R, Balakrishnan G, Barrett A. Multifocal synchronous osteosarcoma: the Scottish Bone Tumor Registry Experience. Med Pediatr Oncol 1993;21: 111-16.

4. Hopper KD, Moser RP, Haseman DB, et al. Osteosarcomatosis. Radiology 1990; 175:223-39

5. Daffner RH, Kennedy SL, Fox KR, et al. Synchronous multicentric osteosarcoma: the case for metastases. Skeletal Radiol 1997;26:569-78.

6. Parham DM, Pratt CB, Parvey LS, Webber BL, Champion J. Childhood multifocal osteosarcoma: clinicopathologic and radiologic correlates. Cancer 1985;55: 2653-8.

7. Shinozaki T, Chigira M, Watanabe H, Kaneko K. Osteosarcoma with multiple skeletal metastases: a case of "nonstochastic" metastasis. Arch Orthop Trauma Surg 1993:112:292-5

8. Rosen G. Preoperative (neoadjuvant) chemotherapy for osteogenic sarcoma: a ten year experience. Orthopaedics 1985;8:659-64.

9. Meyers PA, Heller G, Healey J, et al. Chemotherapy for nonmetastatic osteogenic sarcoma: the Memorial Sloan-Kettering experience. J Clin Oncol 1992;10:5-15.

10. Provisor AJ, Ettinger LJ, Nachman JB, et al. Treatment of nonmetastatic osteosarcoma of the extremity with preoperative and postoperative chemotherapy: a report from the Children's Cancer Group. J Clin Oncol 1997;15:76-84.

11. Fuchs $\mathbf{N}$, Bielack SS, Epler $\mathbf{D}$, et al. Long-term results of the co-operative GermanAustrian-Swiss osteosarcoma study group's protocol COSS-86 of intensive multidrug chemotherapy and surgery for osteosarcoma of the limbs. Ann Col 1998;9:893-9.

12. Saeter G, Wiebe T, Wiklund T, et al. Chemotherapy in osteosarcoma: The Scandinavian Sarcoma Group experience. Acta Orthop Scand Supp/1999;285:74-82.
13. Meyers PA, Heller G, Healey JH, et al. Osteogenic sarcoma with clinically detectable metastases at initial presentation. J Clin Oncol 1993;11:449-53.

14. Thompson RC Jr, Cheng EY, Clohisy DR, et al. Results of treatment for metastatic osteosarcoma with neoadjuvant chemotherapy and surgery. Clin Orthop 2002:397: 240-7.

15. Harris MB, Gieser P, Goorin AM, et al. Treatment of metastatic osteosarcoma at diagnosis: a Pediatric Oncology Group study. J Clin Oncol 1998;16:3641-8.

16. Bacci G, Briccoli A, Rocca M, et al. Neoadjuvant chemotherapy for osteosarcoma of the extremities with metastases at presentation: recent experience at the Rizzoli Institute in 57 patients treated with cisplatin, doxorubicin, and a high dose of methotrexate and ifosfamide. Ann Oncol 2003:14:1126-34.

17. Bacci G, Picci P, Ferrari S, et al. Synchronous multifocal osteosarcoma: results in twelve patients treated with neoadjuvant chemotherapy and simultaneous resection of all involved bones. Ann Oncol 1996; 7:864-6.

18. Longhi A, Fabbri N, Donati $\mathbf{D}$, et al. Neoadjuvant chemotherapy for patients with synchronous multifocal osteosarcoma: results in eleven cases. J Chemother 2001;13: 324-30.

19. Fletcher CDM, Unni KK, Mertens F, eds. Pathology \& genetics: tumours of soft tissue and bone. In: World Health Organization classification of tumours. Lyon: IARC Press, 2002.

20. Bacci G, Briccoli A, Ferrari S, et al. Neoadjuvant chemotherapy for osteosarcoma of the extremity: Iong-term results of the Rizzoli's 4th protocol. Eur J Cancer 2001;37: 2030-9

21. Bacci G, Ferrari S, Longhi A, et al. High dose ifosamide in combination with high dose methotrexate, adriamycin and cisplatin in the neoadjuvant treatment of extremity osteosarcoma: preliminary results of an Italian Sarcoma Group/Scandinavian Sarcoma Group pilot study. J Chemother 2002;14:198-206.

22. Enneking WF, Spanier SS, Goodman MA. A system for the surgical staging of musculoskeletal sarcoma: 1980. Clin Orthop 2003;415:4-18.

23. Picci P, Bacci G, Campanacci M, et al. Histologic evaluation of necrosis in osteosarcoma induced by chemotherapy: regional mapping of viable and nonviable tumor. Cancer 1985:56:1515-21.

24. Silverman G. Multiple osteogenic sarcoma. Arch Pathol 1936;21:88-95.

25. Mahoney JP, Spanier SS, Morris JL. Multifocal osteosarcoma: a case report with review of the literature. Cancer 1979;44:1897-907.

26. Unni KK. Dahlin's bone tumors: general aspects and data on 11087 cases. Fifth ed. Philadelphia: Lippincott-Raven 1996:143-83

27. Campanacci M. Bone and soft tissue tumors. Second ed. Wien: Springer-Verlag, 1999:505.

28. Briccoli A, Rocca M, Salone M, et al. Resection of recurrent pulmonary metastases in patients with osteosarcoma. Cancer 2005;104:1721-5. 